\title{
RATE OF APPROACH TO MINIMA AND SINKS- THE MORSE-SMALE CASE
}

BY

\author{
HELENA S. WISNIEWSKI ${ }^{1}$
}

\begin{abstract}
The dynamical systems herein are Morse-Smale diffeomorphisms and flows on $C^{\infty}$ compact manifolds. We show the asymptotic rate of approach of orbits to the sinks of the systems to be bounded by an expression of the form $K \exp (-D N)$, where $D$ may be any number smaller than $C=\min _{P}\left\{1 / m \log \operatorname{Jac} D_{P} f^{m} \mid W^{u}(P)\right\}$. Here the minimum is taken over all nonsink $P$ in the nonwandering set of $f$, and $m$ is the period of $P$. We extend our theorems to the entire manifold, so that there is no restriction on the location of the initial points of trajectories.
\end{abstract}

1. Introduction. The dynamical systems herein are Morse-Smale diffeomorphisms and flows on compact manifolds of finite dimension. Determining the asymptotic rate of approach of orbits to sinks amounts to comparing the Riemannian measure of the entire manifold to the measure of the set of points whose orbits remain outside a neighborhood of the sinks after $N$ iterations for diffeomorphisms, or time $T$ for flows.

Let $P$ be a fixed point for $f$ which is either a source or a saddle and let $U$ be a neighborhood of $P$ on $M$. If $x \in U$, then unless $x$ is on the stable manifold of $P$, the orbit of $P$ under $f$ will leave $U$. This is simply the familiar fact from the stable manifold theorem that for $U$ small enough $\bigcap_{n=0}^{\infty} f^{-n}(U)$ is the local stable manifold of $P$. The volume lemmas of Bowen and Ruelle [2] and Fried and Shub [5] add to this statement that the measure of $\bigcap_{n=0}^{\infty} f^{-n}(U)$ decays exponentially with the rate. By this we mean that the exponential constant is related to the logarithm of the Jacobian determinant of the unstable part of $f$ at $P$. However, these results are local in nature; they only concern the rate at which orbits leave a neighborhood of $P$. We prove the related global results for all Morse-Smale diffeomorphisms and flows.

Throughout the paper $M$ will be a $C^{\infty}$ compact Riemannian manifold, and $\mu$ will be a measure derived from the Riemannian metric on $M$. For $r>1$, let $\operatorname{Diff}(M)$ be the set of $C^{r}$ diffeomorphisms of $M$. For the definitions of nonwandering point,

Received by the editors June 16, 1981 and, in revised form, July 6, 1983. Portions of this paper were presented at the following meetings: January 1981, AMS 87th Annual Meeting, San Francisco, November 1981, Special Session on Dynamical Systems, AMS 790th Meeting, Austin, October 1982, Special Session in Differential Equations, AMS 797th Meeting, College Park.

1980 Mathematics Subject Classification. Primary 58F09; Secondary 58F15.

Key words and phrases. Dynamical systems, diffeomorphism, Morse-Smale systems, Axiom A systems, no-cycles, transversality, filtration, hyperbolic invariant set, basic set attractor, flow, unstable and stable manifold.

${ }^{1}$ This paper is part of the author's Ph.D. dissertation completed at the Graduate School and University Center of C.U.N.Y., under the direction of Professor Michael Shub. 
stable and unstable manifold, and Morse-Smale diffeomorphism we refer the reader to $[7,10]$. Here we define filtrations.

Definition. Let $f \in \operatorname{Diff}(M)$. A filtration for $f$ is a sequence of compact manifolds with boundary such that

$$
M=M_{k} \supset M_{k-1} \supset \cdots \supset M_{1} \supset M_{0}=\varnothing
$$

$\left(\operatorname{dim} M_{i}=\operatorname{dim} M\right.$, for $\left.i=1, \ldots, k\right)$ and $f\left(M_{k}\right) \subset$ int $M_{k}$. Given a filtration, $K_{i}=$ $\bigcap_{n \in Z} f^{n}\left(M_{i}-\right.$ int $\left.M_{i-1}\right)$ is the maximal invariant set contained in $\left(M_{i}-M_{i-1}\right)$. If $K_{i}=\Omega \cap\left(M_{i}-M_{i-1}\right)$ for all $i$, we say that the filtration is a fine filtration for $f$. Finally, if we are given closed invariant sets which are disjoint $\Lambda_{1}, \ldots, \Lambda_{k}$, we say $M=M_{k} \supset M_{k-1} \supset \ldots \supset M_{1} \supset M_{0}=\varnothing$ is a filtration for $\Lambda_{1}, \ldots, \Lambda_{k}$ if $\Lambda_{i}=K_{i}$.

Definition. $S(i, j, N)=M_{i}-f^{-N}$ (int $\left.M_{j}\right)$.

Given a filtration we may assume without loss of generality that $M_{1}$ contains all the sinks. We will denote $\left(M_{i}-\right.$ int $\left.M_{j}\right)$ by $M[i, j]$.

DEFINITION. Jac $D f^{u} P_{j}=\operatorname{Jac}\left(D f: E_{P_{j}}^{u} \rightarrow E_{P_{j}}^{u}\right)$.

The following theorem is our main result.

THEOREM A. Let $f$ be any Morse-Smale diffeomorphism on $M$. Let l be the least common multiple of the periods of the periodic orbits of $f$. Let $\delta>0$ be given. Then for all $N$, and $i>j$ we have

$$
\mu(S(i, j, N)) \leqslant K(1+\delta)^{N} \exp ((-C / l) N)
$$

with $K>0$ independent of $N$, and

$$
C / l=\operatorname{Min}\left\{1 / m \log \operatorname{Jac} D_{p_{j}} f^{m} \mid W^{u}\left(p_{j}\right)\right\},
$$

where the minimum is taken over all nonsink $p_{j}$ in the nonwandering set for $f, p_{j}$ not a sink and $f^{m} p_{j}=p_{j}$.

The proof of Theorem A consists of 3 parts and is presented in detail in $\$ 4$. The following is a sketch.

(1) First let $g=f^{\prime}$. Then $g$ is a Morse-Smale diffeomorphism with $\Omega(g)=\operatorname{Fix}(g)$. Once the result is obtained for $g$, its extension to $f$ is immediate.

(2) We know from [10] that $g$ has a fine filtration: $M=\bar{M}_{r} \supset \bar{M}_{r-1} \supset \cdots \supset \bar{M}_{1}$ $\supset \bar{M}_{0}=\varnothing$ such that the maximal invariant set contained in $\operatorname{int}\left(\bar{M}_{1}-\bar{M}_{i-1}\right)$ is a fixed point which we label $P_{i}$. Also $P_{1}, P_{2}, \ldots, P_{s}$ for $1 \leqslant s<r$ are sinks. So for simplicity we define a new filtration by

$$
M=M_{r-s+1} \subset M_{r-s} \subset \cdots \subset M_{2} \subset M_{1} \subset M_{0}=\varnothing
$$

where $M_{1}=\bar{M}_{s}, M_{2}=\bar{M}_{s+1}$, etc. Thus all the sinks of $g$ are located in $M_{1}$.

We then make use of

THEOREM B. Let $f$ be a Morse-Smale diffeomorphism on the $C^{\infty}$ compact manifold $M$ with $\Omega(f)=\operatorname{Fix}(f)$. Let $\mu$ be a measure derived from the Riemannian metric on $M$. Let $\delta>0$ be given. Then for each $P_{i} \in \Omega(f), i=s+1, \ldots, r$, there is a compact neighborhood $U\left(P_{i}\right)$ such that with $U_{N}\left(P_{i}\right)=\left\{x \in U\left(P_{i}\right): f^{k}(x) \notin M_{1}\right.$ for $\left.k \leqslant N\right\}$. 
Furthermore

$$
\mu\left(U_{N}\left(P_{i}\right)\right) \leqslant K(1+\delta)^{N} \exp (-C N),
$$

where $K>0$ is independent of $N$ and $\exp (C)=\min _{j=s+1, \ldots, i}\left\{\operatorname{Jac} D f^{u} P_{j}\right\}$.

This means that $\mu\left(U_{N}\left(P_{i}\right)\right)$ decays exponentially with $N$.

We note that Theorem B establishes Theorem A in the special case that the trajectories are constrained to begin in a neighborhood of a periodic point. Theorem $\mathrm{B}$ and the technical lemmas needed are proven in $\$ 3$.

(3) Next consider: $S(i, 1, N)=M_{i}-f^{-N}$ (int $\left.M_{1}\right)$. We write

$$
S(i, 1, N)=S(i-1,1, N) \cup S(i, i-1, N) \cup A_{N},
$$

where $A_{N}$ is the set of points in $M[i, i-1]$ whose trajectories enter $M_{i-1}$ but do not reach $M_{1}$ through $N$ iterations. We proceed by induction so that the volume estimate for $S(i-1,1, N)$ is assumed known. By the filtration we show that the measure of $S(i, i-1, N)$ is related by bounded factors to the measure of $U_{N}\left(P_{i}\right)$. Again by the filtration we show that $A_{N}$ subdivides further into two parts-one whose orbits pass to $M_{i-1}$ in a bounded number of iterations and one where orbits enter $U\left(P_{i}\right)$ again in bounded iterations. The measure of the first is thus related by bounded factors to $\mu(S(i-1,1, N))$ and the measure of the second is related by bounded factors to $\mu\left(U_{N}\left(P_{i}\right)\right)$. Thus we obtain the result for $\mu(S(i, 1, N))$. Since $S(1, j, N) \subset S(i, 1, N)$ the proof is completed.

In $\$ 2$ we begin the body of the paper with an example of a Morse-Smale diffeomorphism whose rate of volume decay is exactly that given in our theorems.

$\$ 5$ concludes the paper with the proof that our results carry over to the case of Morse-Smale flows.

Without the assumption of transversality, we found the exponential constant to be more complicated and to yield a slower overall rate of decay. This is the case for general $C^{2}$ Axiom A systems with no cycles. For Axiom A systems we found the exponential constant relates to the topological pressure of $f$. We refer the interested reader to our paper [11].

This manuscript is part of my doctoral dissertation. I would like to acknowledge my advisor Professor Michael Shub for presenting me with this topic and for his valuable suggestions. I wish to thank Professor Edgar Feldman for providing me with funding, through his National Science Foundation Research Grant. I also wish to thank Professor Richard Sacksteder for many helpful conversations and Diego Benardette for his proofreading.

2. An example with transversal intersection. Consider the torus $T^{2} \subset R^{3}$ which is tilted back with respect to the horizontal plane. Let the gradient field on it be of the form

$$
\dot{X}=-\operatorname{grad}(h),
$$

where $h$ is the height function of points in relation to the horizontal plane. So the flow is downward. Fix time $t$ to $\tau$, and consider the diffeomorphism $x_{\tau}$. Now consider a diffeomorphism $g$ described as follows: let $g=\mathrm{id}$ on the torus except in a 
small band. In this band $g$ moves points along their level curves. $g$ rotates the level curves in the following manner: the uppermost curve rotates 0 radians, the lowermost rotates $2 \pi$ radians, and the curves in between rotate from 0 to $2 \pi$ going down the band. Set $h=X \circ g$. The effect of $h$ is to push $g$ down causing repetition of the pattern resulting in the transversal intersection of stable and unstable manifolds (Figure 1).

FIGURE 1

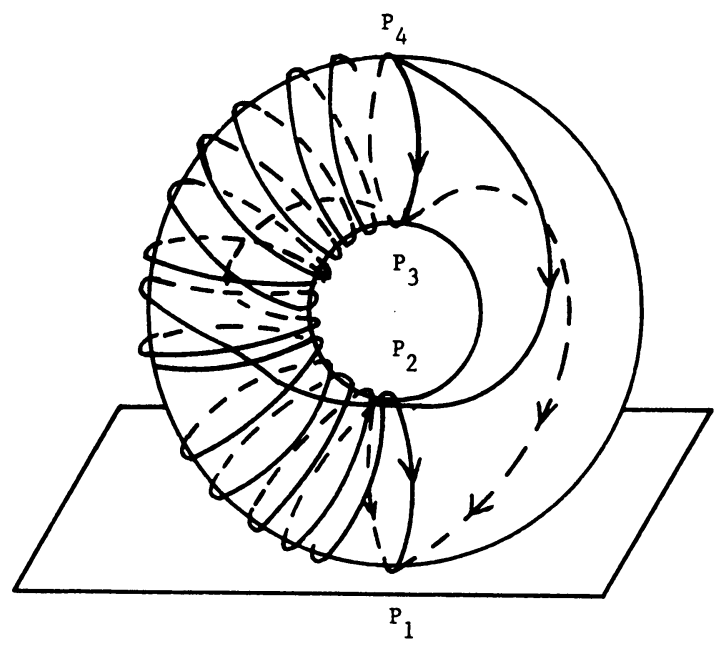

The intersection between $P_{3}$ and $P_{2}$ is illustrated in Figure 2. We note that in the figures, $P_{4}$ is the source, $P_{3}$ and $P_{2}$ are the saddles, and $P_{1}$ is the sink.

In Figure 2 there are an infinite number of such intersections tending forward to $P_{2}$. The $\lambda$ Lemma of Palis [6] tells us that $W^{u}\left(P_{3}\right)$ in a neighborhood of $P_{2}$ becomes close to $W^{u}\left(P_{2}\right)$ in both distance and slope. In fact, it contains $W^{u}\left(P_{2}\right)$ in its closure.

Next in linearized neighborhoods of $P_{3}$ and $P_{2}$ the local diffeomorphisms can be given by

$$
\begin{aligned}
& X=X_{0} \exp (\gamma), \quad X=X_{0} \exp (-\gamma), \\
& Y=Y_{0} \exp (-\alpha), \quad Y=Y_{0} \exp (\beta),
\end{aligned}
$$

respectively. The logarithms of the eigenvalues are $\gamma,-\alpha,-\gamma, \beta$. For sets moving from $P_{3}$ to $P_{2}$, the transversality property causes the two unstable directions to become aligned. This is shown in Figure 2, where we only consider one point of transversal intersection outside the nieghborhoods for clarity.

By the transversality property the height of the set which comes into the neighborhood of $P_{2}$ is independent of the number of iterations for which it stays in a neighborhood of $P_{3}$. So only $W^{u}\left(P_{2}\right)$ influences the set in the neighborhood.

Using the diffeomorphisms given in $(* *)$ we show that the area of the set whose orbits remain in the neighborhood of $P_{3}$ for exactly $n$ iterations and in the neighborhood of $P_{2}$ for at least $m$ iterations equals $4 \varepsilon^{2} \exp (-\beta m) \exp (-\gamma n)$. 
FIGURE 2

$$
D=[\varepsilon \exp (-\gamma), \varepsilon] \times[-\varepsilon, \varepsilon]
$$

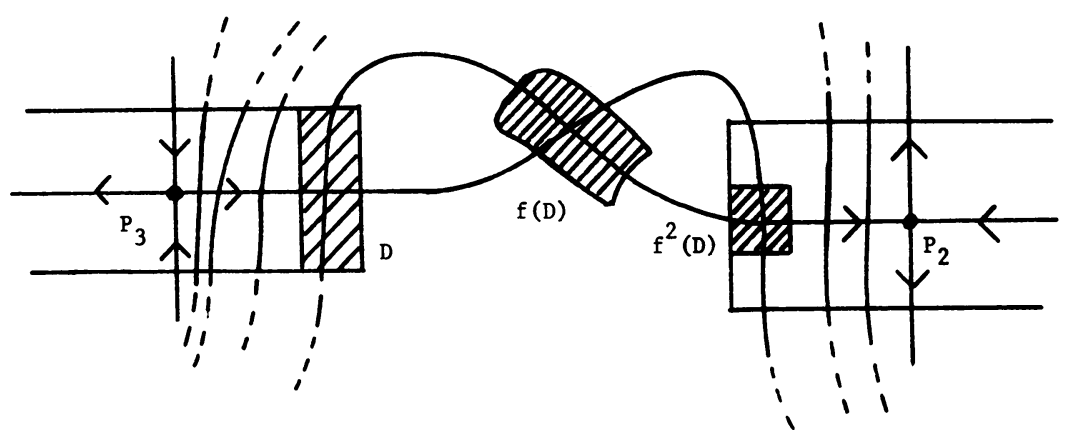

To see how this estimate is obtained consider the following illustrations. First consider the linearized neighborhood of $P_{3}$, in Figure 3.

Figure 3

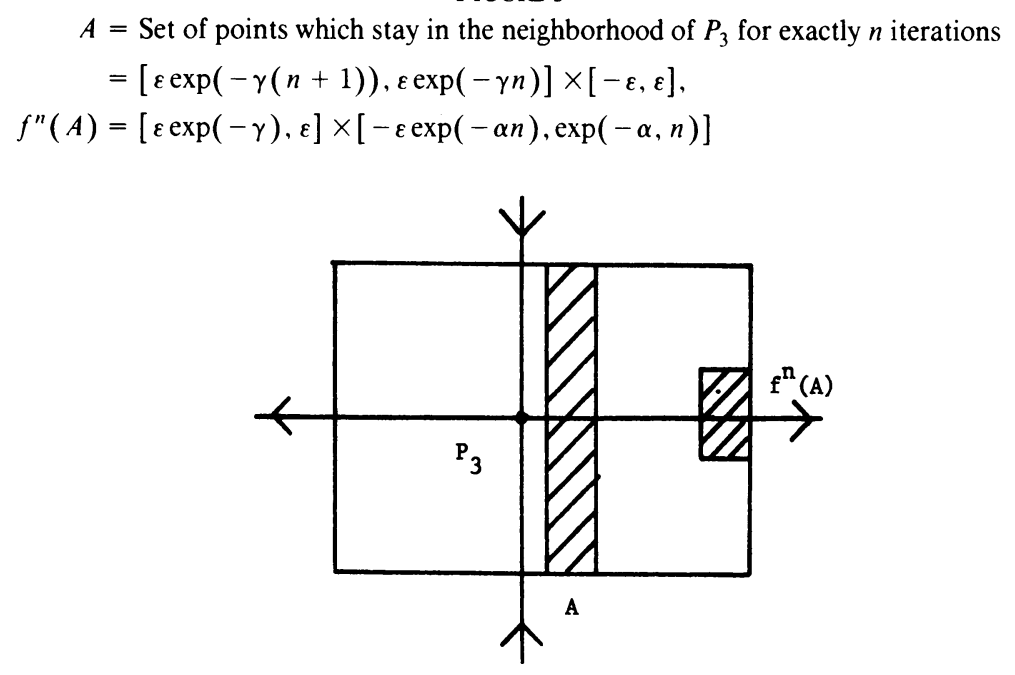

FIGURE 4

$B=f^{n+1}(A)$. The height of $B=$ width $A=\varepsilon(1-\exp (-\gamma))$. Due to the transversality condition, the height is independent of the number of iterations the set was at $P_{3}$.

$$
B=[-\varepsilon,-\varepsilon \exp (-\gamma)] \times[-(\varepsilon / 2)(1-\exp (-\gamma), \varepsilon / 2)] .
$$

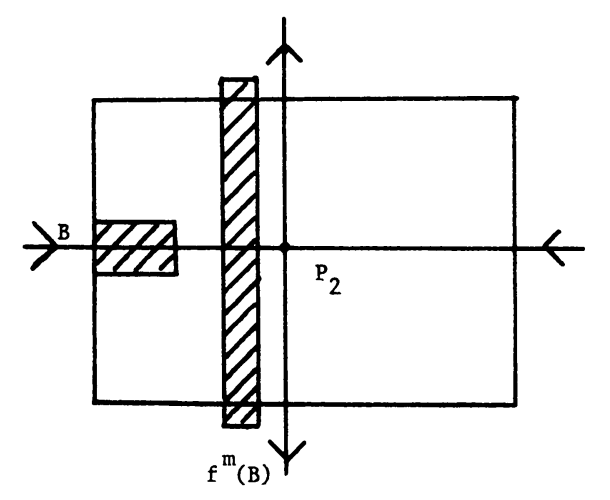


FIGURE 5

$B^{\prime}=$ Set of points which stay in the neighborhood of $P_{2}$ for $m$ iterations. It is denoted by the shaded strip.

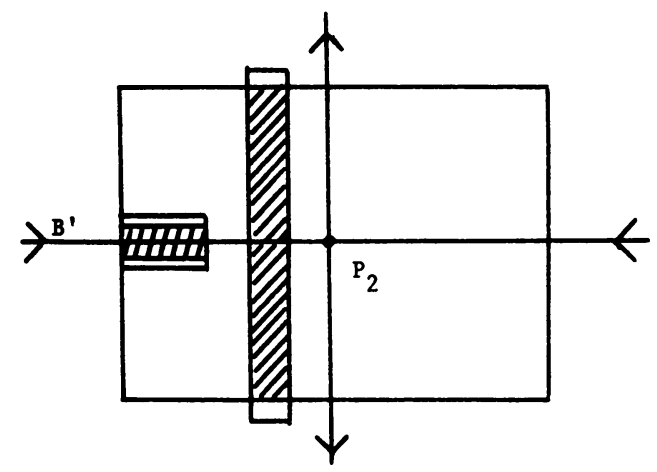

FIGURE 6

Translate the set $B^{\prime}$ back to $P_{3}$. "Go back" $n$ iterations at $P_{3}$.

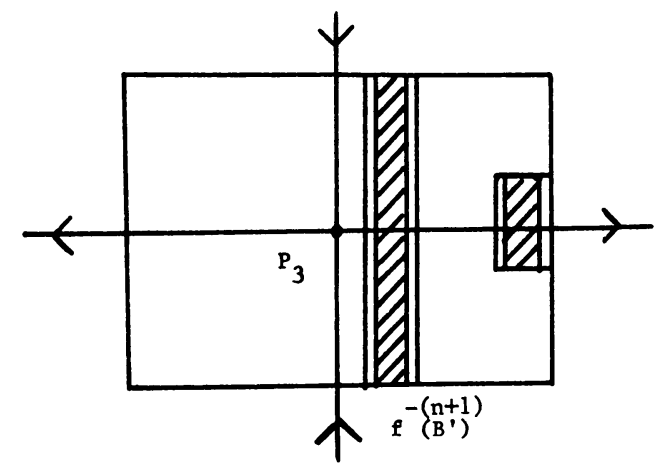

Let $E=\left\{f^{-(n+1)}\right\}\left(B^{\prime}\right)$. The width of $E=2 \varepsilon \exp (-\beta m) \exp (-\gamma n)$. The area of $E=$ $4 \varepsilon^{2} \exp (-\beta m) \exp (-\gamma n)$. Take $\beta=\gamma$ and call it $C$.

Furthermore, to obtain the area of the set in the neighborhood of $P_{3}$ whose orbits do not leave the neighborhoods of $P_{2}$ or $P_{3}$ through $N$ iterations, sum over $n$ and $m$ in the relation $N=n+m+1$. Considering the number of ways to add $n+m$ we have the area $=\sum_{n=0}^{N} 4 \varepsilon^{2} \exp (C) \exp (-C N)$, so we obtain that the area $=$ $4 \varepsilon^{2}(\exp C)(N+1) \exp (-C N)$. We note that this example is a Morse-Smale diffeomorphism.

3. The Proof of Theorem B. We prove Theorem B in two stages. First we consider submanifolds of $M$ in a neighborhood $U(P)$ of $P \in \Omega(f)$ which are of the same dimension as $W^{u}(P)$ and $C^{1}$ close to $W^{u}(P)$, showing that the volume of these submanifolds in $U_{N}(P)$ decays exponentially. Secondly, we cover $U_{N}(P)$ by a $C^{1}$ system of such manifolds and use Fubini's Theorem to arrive at our result for $U_{N}(P)$. 
For $P \in \Omega(f)$ we know that $T_{P} M$ splits into the direct sum decomposition

$$
T_{P} M=E^{s} \oplus E^{u},
$$

with stable $\left(E^{s}\right)$ and unstable $\left(E^{u}\right)$ subspaces. Let $($,$) be a Riemannian metric on$ $M$ adapted to $f$; that is for $v=\left(v_{s}, v_{u}\right) \in T_{P} M$,

$$
\left\|D_{P} f\left(v_{s}\right)\right\| \leqslant \lambda\left\|v_{s}\right\| \text { and }\left\|D_{P} f\left(v_{u}\right)\right\| \geqslant \lambda^{-1}\left\|v_{u}\right\|
$$

when $0<\lambda<1$ is independent of $P$ and $\|w\|^{2}=(w, w)$.

We introduce a modified metric $\langle$,$\rangle on M$ by $\langle v, w\rangle=\left(v_{s}, w_{s}\right)+\left(v_{u}, w_{u}\right)$ and denote the stable and unstable seminorms $\left(v_{s}, v_{s}\right)^{1 / 2}$ and $\left(v_{u}, v_{u}\right)^{1 / 2}$ by $\|v\|_{s}$ and $\|v\|_{u}$. Then the norm defined by $\langle v, v\rangle^{1 / 2}$ is

$$
\|v\|=\left(\|v\|_{s}^{2}+\|v\|_{u}^{2}\right)^{1 / 2} .
$$

For a subspace $V \subset T_{P} M, v(\varepsilon) \equiv\{v \in V:\|v\| \leqslant \varepsilon\}$. For small $\varepsilon>0$ we can find a $C^{1}$ chart $\phi: T_{P} M(\varepsilon) \rightarrow M$ such that:

(1) $\phi(0)=P$ and $D_{0} \phi: T_{P} M \rightarrow T_{P} M$ is the identity;

(2) $\phi\left(E^{s}(\varepsilon)\right) \subset W_{P}^{s}$; and

(3) $\phi\left(E^{u}(\varepsilon)\right) \subset W_{P}^{u}$

where $W_{P}^{s}$ and $W_{P}^{u}$ are the stable and unstable manifolds at $P$. The map $F=$ $\phi^{-1} \circ f \circ \phi$ represents $f$ at $P$ in this coordinate system (Bowen and Ruelle [2] and Fried and Shub [5]). Such a chart can be chosen for each element of $\Omega(f)$ and we assume that $\varepsilon$ suffices for all $P \in \Omega(f)$.

In Proposition 1 the slope of a smooth manifold $N$ of $T_{i} M(\varepsilon)$ with $\operatorname{dim}(N) \leqslant$ $\operatorname{dim}\left(E^{u}\right)$ at a point $n \in N$ refers to the supremum of the quotients $\left\|v^{s}\right\| /\left\|v^{u}\right\|$, taken over all tangent vectors $v=\left(v^{s}, v^{u}\right)$ to $N$ at $n$. To say that $N$ has slope less than or equal to $\omega$ relative to $E_{i}^{u}$ means that for all $n \in N$, the slope of $N$ at $n$ is less than or equal to $\omega$.

Definition. $B_{i}(\varepsilon)=\left\{x \in M: d\left(x, P_{i}\right) \leqslant \varepsilon\right\}$.

In the remainder of the paper, for $P_{i} \in \Omega(f)$, let $T_{i} M=T_{P_{i}} M, E_{i}^{s}=E_{P_{i}}^{s}$ and $E_{i}^{u}=E_{P_{i}}^{u}$.

Definition. The graph transform $\Gamma_{i}$ defined on $C^{1}$ functions $g$ from $E_{i}^{u}\left(\varepsilon_{i}\right)$ to $E_{i}^{s}\left(\varepsilon_{i}\right)$ is defined by graph $\Gamma_{i}(g)=F_{i}$ (graph $g$ ) with $F_{i}=\phi_{i}^{-1} \circ f \circ \phi_{i}$.

We assume that $\varepsilon$ is small enough so that we have $C^{1}$ charts as specified and graph transforms.

Proposition 1. For $\varepsilon, \delta>0$ and $0<\omega$ finite there exist constants $\varepsilon_{i}, \omega_{i}, i=2, \ldots, r$, such that

(1) $\varepsilon_{i} \leqslant \varepsilon$

(2) $\omega_{j} \geqslant \omega_{i} / 3$ for $i<j$.

(3) If $L \in \operatorname{Hom}\left(E_{i}^{u}, E_{i}^{s}\right),\|L\| \leqslant \omega_{i}$, and $v \in T_{i} M\left(\varepsilon_{i}\right)$, then

$$
\operatorname{Jac} D_{v}(F \mid v+\operatorname{graph} L) \geqslant \operatorname{Jac} D_{0}\left(F \mid E_{i}^{u}\right) /(1+\delta) .
$$

(4) For g a $C^{1}$ function from $E_{i}^{u}\left(\varepsilon_{i}\right)$ to $E_{i}^{s}\left(\varepsilon_{i}\right)$, slope $g \leqslant \omega_{i}$ implies that slope graph $\Gamma_{i}(g) \leqslant \omega$. 
(5) $T_{i} M\left(\varepsilon_{i}\right)$ is contained in a neighborhood $V_{i}$ of the origin in $T_{i} M$ such that for all $P_{k} \in \Omega(f)$ either $W_{k}^{u} \cap W_{i}^{s}=\varnothing$ or $\phi_{i}^{-1}\left(\left(W_{k}^{u}\right) \cap T_{i} M\left(\varepsilon_{i}\right)\right) C^{1}$-fibers over the intersection of $\phi_{i}^{-1}\left(W_{k}^{u}\right)$ with $E_{i}^{s}$ in $V_{i}$, each fiber having dimension equal to the dimension of $W_{i}^{u}$ and slope no greater than $\omega_{i} / 3$.

(6) For $i>j$, if $W_{i}^{u} \cap W_{j}^{s} \neq \varnothing$, then given any $C^{1}$ function $h$ of $E_{i}^{u}\left(\varepsilon_{i}\right)$ to $E_{i}^{s}\left(\varepsilon_{i}\right)$ for which slope graph $h \leqslant \omega_{i}$ and $\left(f^{n} \phi_{i}\right.$ graph $\left.h\right) \cap B_{j}\left(\varepsilon_{j}\right) \neq \varnothing$, then $\phi_{j}^{-1} f^{n} \phi_{i}(\operatorname{graph} h)$ $C^{1}$-fibers over $\left(\phi_{j}^{-1} W_{i}^{u}\right) \cap E_{j}^{s}$, with the fibers having slope less than or equal to $\psi_{j}$.

Proof. Before beginning the proof we consider the following definition and lemma.

Definition [6]. Let $f \in \operatorname{Diff}(M)$ and $P$ be a hyperbolic fixed point of $f$. We denote by $L S(P)$ and $L U(P)$ the local stable and unstable manifold of $P$, for $s=$ dimension $L S(P)$. Let $B^{s}$ be a cell neighborhood of $P$ in $L S(P)$, such that $f\left(\partial B^{s}\right) \subset$ int $B^{s}$. The existence of such a cell $B^{s}$ follows from the fact that $f \mid L S(P)$ is a contraction. The embedded annulus in $L S(P)$ whose boundaries are $B^{s}, f\left(\partial B^{s}\right)$, is called a fundamental domain $G^{s}(P)$ of $W^{s}(P)$.

We note that $W^{s}(P)=\bigcup_{n \in \mathbf{Z}} f^{n}\left(G^{s}(P)\right) \cup P$. Any neighborhood $N^{s}(P)$ of $G^{s}(P)$ in $M$, disjoint from $L U(P)$, is called a fundamental neighborhood associated with $W^{s}(P)$.

Dually we can define $G^{u}(P)$ and $N^{u}(P)$.

Lemma (1.11 of [6]). Fix a cell neighborhood $B^{r}$ of $P$ in $L U(P)$. There exists a neighborhood $V$ or $P$, such that $W^{u}\left(P_{i}\right) \cap V=\varnothing$, or $W^{u}\left(P_{i}\right) \cap V$ is an r-cell fiberbundle over $W^{u}\left(P_{i}\right) \cap V \cap L S(P)$ with the fibers $C^{1}$ close to $B^{r} .\left(P_{i} \in \Omega(f)\right.$.)

Moreover, the fibering can be chosen to be $C^{1}$.

We now begin the proof of Proposition 1. For simplicity we assume that $f$ has one sink. The proof is by induction using [5]. Let $i=2$. Take $\bar{\varepsilon}_{2} \leqslant \varepsilon$ and $0<\omega_{2}<\omega$ so that $(*)$ holds in $T_{2} M\left(\bar{\varepsilon}_{2}\right)$. Furthermore take $\varepsilon_{2}^{\prime} \leqslant \bar{\varepsilon}_{2}$ so that if $g: E_{2}^{u}\left(\varepsilon_{2}^{\prime}\right) \rightarrow E_{2}^{s}\left(\varepsilon_{2}^{\prime}\right)$ is a $C^{1}$ function with slope $\leqslant \omega_{2}$ then the graph transform of $g$ by $f, \Gamma_{2} g$, also has slope $\leqslant \omega$ (Fried and Shub [5]). This satisfies (1)-(4) of the proposition, for $i=2$.

Next we consider (5). By the preceding results we can take $\varepsilon_{2} \leqslant \varepsilon_{2}^{\prime}$ so that $T_{2} M\left(\varepsilon_{2}\right)$ is contained within the neighborhood $V$ of $P_{2}$ guaranteed by Lemma 1.11 of [6] for $P_{i} \in \Omega(f)$, with $r=\operatorname{dim} L U(P)$. Thus, if $\phi_{2}^{-1}\left(W_{k}^{u}\right) \cap T_{2} M\left(\varepsilon_{2}\right)$ is not empty we get that it $C^{1}$-fibers over its intersection with $E_{2}^{s}$, with all of its fibers having slope no greater than $\omega_{2} / 3$.

By taking $T_{2} M\left(\varepsilon_{2}\right)$ properly contained in $V$ we can get the $C^{1}$ fiber structure. Thus (5) is satisfied, and this completes the proof for $i=2$, since $P_{1}$ is the sink.

Assume that $\varepsilon_{j}, \omega_{j}$ have been chosen as to satisfy (1)-(6) for $j=1, \ldots, i-1<r$. We show how to choose $\varepsilon_{i}$ and $\omega_{i}$. First take $\bar{\varepsilon}_{i} \leqslant \varepsilon$ and $0<\bar{\omega}_{i} \leqslant \omega<1$ so that the Jacobian inequality (*) of Proposition 1 holds. Then take $\omega_{i} \leqslant \bar{\omega}_{i}$ and $\omega_{i} \leqslant \omega_{i-1} / 3$ (this implies $\omega_{i} \leqslant \omega_{j} / 3$ for $j<1$ ). Next choose $\varepsilon_{i}^{\prime} \leqslant \bar{\varepsilon}_{i}$ so that the graph transform $\Gamma_{i}$ satisfies $\|D h\| \leqslant \omega_{i}$. Then $\left\|D \Gamma_{i}(h)\right\| \leqslant \omega$ for $h \in C^{1}, h: E_{i}^{u}\left(\varepsilon_{i}^{\prime}\right) \rightarrow E_{i}^{s}\left(\varepsilon_{i}^{\prime}\right)$ (Fried and Shub [5]).

For the next step observe that if any orbit of $f$ leaves the neighborhood $\phi_{i}\left(T_{i} M\left(\varepsilon_{i}^{\prime}\right)\right)$ of $P_{i}$ on $M$ then there is a bounded numoer of iterations $N_{i}$ by which (1) the orbit is 
in a neighborhood of the sink $P_{1}$ or (2) the orbit has entered (and possibly left) one of the neighborhoods $\phi_{j}\left(T_{j} M\left(\varepsilon_{j}\right)\right)$. Thus we suppose that $N$ is a $C^{1}$ submanifold of $T_{i} M\left(\varepsilon_{i}^{\prime}\right)$ and that $f^{k} \phi_{i} N \cap B_{j}(\varepsilon) \neq \varnothing$, for some $k \leqslant N_{i}$. By taking $N$ in a tubular neighborhood of $E_{i}^{u}\left(\varepsilon_{i}^{\prime}\right)$ we get that $\phi_{j}^{-1} f^{k} \phi_{i} N \cap T_{j} M\left(\varepsilon_{j}\right)$ inherits a $C^{1}$ fiber structure over its intersection with $E_{j}^{s}$ in $V_{j}$ from $\phi_{j}^{-1} W_{i}^{u}$.

If we choose the width of the tubular neighborhood of $E_{i}^{u}\left(\varepsilon_{i}^{\prime}\right)$ small enough, say $\varepsilon_{i}^{*}$, we can insure that since $k \leqslant N_{i}$, that the slope of the fibers of $\phi_{j}^{-1} f^{k} \phi_{i} N-$ $\phi_{j}^{-1}\left(W_{i}^{u}\right)$ relative to $E_{j}^{u}$ is no more than $\omega_{i}$ larger than the slope of $N$ relative to $E_{i}^{u}$. Because we are assuming via the induction hypothesis that the slope of $\phi_{i}^{-1}\left(W_{i}^{u}\right)$ relative to $E_{j}^{u}$ is not larger than $\omega_{j} / 3$, the slopes of the fibers of $\phi_{j}^{-1} f^{k} \phi_{i} N$, relative to $E_{j}^{u}$ is bounded by $\omega_{j} / 3+\omega_{i}+$ (slope $N$ relative $E_{i}^{u}$ ). Beginning with an $N$ with slope $\omega_{i}$, relative to $E_{i}^{u}$, gives that the slope of the fibers of $\phi_{j}^{-1} f^{k} \phi_{i} N$, relative to $E_{j}^{u}$, is less than or equal to $\omega_{j} / 3+2 \omega_{i} \leqslant \omega_{j}$. Using $\varepsilon_{i}^{*}$ for the neighborhood of $P_{i}$ in $T_{i} M$ satisfies (6). So we only need to prove (5). But here we argue as for $i=2$ to get $\varepsilon_{i} \leqslant \varepsilon_{i}^{*}$ so that (5) is satisfied.

Thus the $\varepsilon_{i}, \omega_{i}$ have been chosen as to satisfy (1)-(6), and the proof of Proposition 1 is complete.

Proof of Theorem B. For simplicity assume that there is exactly one sink. We note that the following $T_{i} M(\varepsilon)$ is defined in terms of the norm $\|v\|=$ $\max \left\{\|v\|_{s},\|v\|_{u}\right\}$.

Given $\delta>0$, let $\delta^{\prime}<\delta$. Then we can find for all $i=2, \ldots, r$ a pair $\left(\varepsilon_{i}, \omega_{i}\right)$ which satisfy (1)-(6) of Proposition 1 with $\delta^{\prime}$ in place of $\delta$.

Take $\varepsilon>0$ such that $\varepsilon<\min \varepsilon_{i}$ and consider the compact neighborhood of 0 in $T_{i} M, \operatorname{cl} T_{i} M(\varepsilon)=\operatorname{cl}\left(E_{i}^{u}(\varepsilon)+E_{i}^{s}(\varepsilon)\right)$. Then take $U\left(P_{i}\right)=\phi_{i}\left(\operatorname{cl} T_{i} M(\varepsilon)\right)$. These are our neighborhoods of the fixed points. Fix $i$ and let $S_{N}=\phi_{i}^{-1}\left(U_{N}\left(P_{i}\right)\right) ; S_{N} \subset$ cl $T_{i} M(\varepsilon)$.

We prove that $\nu\left(S_{N}\right) \leqslant K(1+\delta)^{N} \exp (-C N)$, where $\nu$ is the ordinary Lebesgue measure in $T_{i} M$. Passing back to the measure $\mu$ on $M$ involves only multiplication by constants bounded away from 0 and $\infty$.

For $v \in \mathrm{cl} E_{i}^{s}(\varepsilon)$ consider the linear variety $H_{v}=\left\{(u, v) \in T_{i} M: u \in E_{i}^{u}\right\}$. Consider the subgraphs $S_{N} \cap H_{v}$. These are compact sets which cover $S_{N}$ in the sense that $S_{N}=\bigcup_{v \in E_{i(\varepsilon)}^{s}}\left[S_{N} \cap H_{v}\right]$.

We appeal to Lemma 2 in order to get an area estimate for the subgraphs $\left(S_{N} \cap H_{v}\right)$.

LEMMA 2. For $i \geqslant 2$, if $h$ : $\mathrm{cl} E^{u}\left(\varepsilon_{i}\right) \rightarrow E^{s}\left(\varepsilon_{i}\right)$ is a $C^{1}$ function with $\|D h\| \leqslant \omega_{i}$, then

$$
\operatorname{area}\left(\operatorname{graph} h \cap S_{N}\right) \leqslant K(1+\delta)^{N} \exp (-C N),
$$

where $K>0, \exp (C)=\min _{2 \leqslant j \leqslant i}\left\{\operatorname{Jac} D f^{\prime}\left(P_{j}\right)\right\}$, and area refers to the induced $r$ dimensional measure along graph $h$, where $r=\operatorname{dimension}\left(E^{u}\right)$.

Proof. The area measures are induced by the Riemannian metric induced by the inner product $\langle v, w\rangle=\left(v^{u}, w^{u}\right)+\left(v^{s}, w^{s}\right)$. The proof is by induction on $i$. If $i=2$, we argue as in [5]. If $x \in S_{N}$, then $F^{k} x \in \operatorname{cl} T_{2} M(\varepsilon)$ for $k=0, \ldots, N-l$, where $l$ is a fixed integer representing the maximum number of iterations for an orbit leaving 
$U\left(P_{2}\right)$ to enter $M_{1}$, the neighborhood of the $\operatorname{sink}(\mathrm{s})$ on $M$ (recall $\left.F=\phi_{i}^{-1} \circ f \circ \phi_{2}\right)$. The same statement thus applies to $F^{k}$ (graph $h \cap S_{N}$ ). Moreover we know that successive iterates of (graph $h \cap S_{N}$ ) are themselves graphs of $C^{1}$ functions of $\operatorname{cl} E_{2}^{u}(\varepsilon)$ to $\mathrm{cl} E_{2}^{s}(\varepsilon)$ with slope $\leqslant \omega_{2}$. This is because $\varepsilon$ was chosen to insure that the graph transform of $h, \Gamma_{2} h$, is a $C^{1}$ function with slope $\leqslant \omega_{2}$.

Thus $F^{N-1}$ (graph $h \cap S_{N}$ ) is indeed the graph of a $C^{1}$ function of cl $E^{u}\left(\varepsilon_{2}\right)$ into $\operatorname{cl} E^{s}\left(\varepsilon_{2}\right)$ with slope $\leqslant \omega_{i}$ so that

(I) $\operatorname{area}\left(F^{N-I}\left(\right.\right.$ graph $\left.\left.h \cap S_{N}\right)\right) \leqslant K$, where $K$ is a constant depending only on $\varepsilon$ and $\omega_{2}$. Moreover,

$$
K \geqslant \int_{\text {graph } h \cap S_{N}} \operatorname{Jac} D_{v}\left(F^{N-l} \mid v+E_{2}^{u}\right) d \mu_{r}
$$

But the Jacobian inequality (*) holds in cl $T_{2} M(\varepsilon)$. That is if $L \in \operatorname{Hom}\left(E_{2}^{u}, E_{2}^{s}\right)$ with $\|D L\| \leqslant \omega_{2}$ and $v \in \operatorname{cl} T_{2} M(\varepsilon)$ we have

$$
\operatorname{Jac} D_{v}(F \mid v+\operatorname{graph} L) \geqslant\left(1+\delta^{\prime}\right)^{-1} \operatorname{Jac} D_{0}\left(F \mid E_{2}^{u}\right) \text {. }
$$

Here $0<\delta^{\prime}<\delta$ is the constant in the proof of Theorem B.

We apply this inequality repeatedly to get from (I) the following:

$$
\left.K \geqslant\left(1+\delta^{\prime}\right)^{-N+l} \operatorname{Jac} D_{0}\left(F^{N-l} \mid E_{2}^{u}\right) \text { area(graph } h \cap S_{N}\right)
$$

so that

$$
\operatorname{area}\left(\operatorname{graph} h \cap S_{N}\right) \leqslant K\left(1+\delta^{\prime}\right)^{N-l}\left(\operatorname{Jac} D_{0}\left(F^{N-l} \mid E_{2}^{u}\right)\right)^{-1} \text {. }
$$

Now since $f$ is assumed to have only fixed points,

$$
\operatorname{Jac} D_{0}\left(F^{N-l} \mid E_{2}^{u}\right)=\operatorname{Jac} D_{0}\left(F \mid E_{2}^{u}\right)^{N-l} \text {. }
$$

Then by redefining $K$,

$$
\operatorname{area}\left(\operatorname{graph} h \cap S_{N}\right) \leqslant K\left(1+\delta^{\prime}\right)^{N}\left(\operatorname{Jac} D_{0}\left(F \mid E_{2}^{u}\right)\right)^{-N} .
$$

By observing that the properties of $\phi_{2}$ allow us to show that $\operatorname{Jac} D f^{u}\left(P_{2}\right)=$ Jac $D_{0}\left(F \mid E_{2}^{u}\right)$, we are done with the $i=2$ case.

Now assume that the result has been proven for all $i<k$, where $k>2$ and prove it holds for graphs in $\operatorname{cl} T_{k} M(\varepsilon)$. Here the situation for (graph $h \cap S_{N}$ ) is different because $x \in S_{N}$ does not imply that $F^{N-l}(x) \in \operatorname{cl} T_{k} M(\varepsilon)$, where $l$ is some fixed integer. In fact on $M$ an orbit beginning in $U\left(P_{k}\right)$ may leave $U\left(P_{k}\right)$ and enter some $U\left(P_{j}\right)$ before reaching $V$. Therefore we must consider $N+1$ separate subgraphs of (graph $h \cap S_{N}$ ). We let $B_{m}$ be that subgraph of (graph $h \cap S_{N}$ ) for which $F^{m+1} B_{m} \cap$ $\operatorname{cl} T_{k} M(\varepsilon)=\varnothing$, and $F^{m} B_{m} \subset \operatorname{cl} T_{k} M(\varepsilon)$ for $0 \leqslant k \leqslant m$ (i.e. $B_{m}$ leaves $T_{k} M(\varepsilon)$ at exactly the $(m+1)$ iteration). Thus (graph $\left.h \cap S_{N}\right)-E_{k}^{s}=\bigcup_{m=0}^{\infty} B_{m}$ where the union is disjoint. We shall modify $B_{m}$ slightly, replacing $B_{m}$ by $\mathrm{cl} B_{m}$. Then $\operatorname{graph}\left(h \cap S_{N}\right) \subset \cup_{m=0}^{\infty} B_{m}$ with the union no longer disjoint but consisting of compact sets. Furthermore we only need to consider a finite union

$$
\text { (graph } \left.h \cap S_{N}\right) \subset A \cup B_{0} \cup B_{1} \cup \cdots B_{N-1}
$$

where $A$ is the closure of that portion of graph $\left(h \cap S_{N}\right)$ which remains in $\operatorname{cl} T_{k} M(\varepsilon)$ for at least $N$ iterations. Since all these sets are disjoint except on their boundaries 
we have

$$
\operatorname{area}\left(\operatorname{graph} h \cap S_{N}\right)=\operatorname{area}(A)+\sum_{m=0}^{N-1} \operatorname{area}\left(B_{m}\right) \text {. }
$$

Furthermore we know by the arguments used in the $i=2$ case that

$$
\operatorname{area}(A) \leqslant K\left(1+\delta^{\prime}\right)^{N} \exp \left(-C_{k} N\right),
$$

and also that

$$
\operatorname{area}\left(B_{m}\right) \leqslant\left(1+\delta^{\prime}\right)^{m} \operatorname{area}\left(F^{m} B_{m}\right) \exp \left(-C_{k} m\right),
$$

where $C_{k}=\operatorname{Jac} D f^{u}\left(P_{k}\right)$. Consider $\operatorname{area}\left(F^{m} B_{m}\right)$. The compact set $\phi_{k} F^{m} B_{m} \subset U\left(P_{k}\right)$ passes out of $U\left(P_{k}\right)$ on the next iteration, that is $f\left(\phi_{k} F^{m} B_{m}\right) \cap \operatorname{int} U\left(P_{k}\right)=\varnothing$. Subgraphs of $\phi_{k} F^{m} B_{m}$ may pass under further iterations of $f$ to any $U\left(P_{j}\right)$ for $j<k$ or to $M_{1}$. However, since $\phi_{k} B_{m} \subset U_{N}\left(P_{k}\right)$ any subgraph passing to $M_{1}$ must be in a $B_{m}$ with $m \leqslant N-l$ for a fixed integer $l$. To define the subgraph of $\phi_{k} F^{m} B_{k}$ we perform the following procedure. $\phi_{k} F^{m} B_{m}$ is contained in the closed fundamental neighborhood (associated to $W_{k}^{u}$ ) given by $D_{K}=U\left(P_{k}\right)-$ int $f^{-1} U\left(P_{k}\right)$. Consider $D_{k} \cap \bigcup_{n=1}^{\infty} f^{-n}\left(U\left(P_{k-1}\right)\right)$; this is in fact equal to $D_{k} \cap \bigcup_{n=1}^{l_{k-1}} f^{-n}\left(U\left(P_{k-1}\right)\right)$ for some fixed $l_{k-1}$ representing the maximum number of iterations for an orbit of $D_{k}$ to enter $U\left(P_{k-1}\right)$ for the first time. Hence the intersection of $D_{k}$ with inverse images of $U\left(P_{k-1}\right)$ is compact. We also know that the subgraph of $\phi_{k} F^{m} B_{m}$ in this intersection is compact.

Now the iterates of $\phi_{k} F^{m} B_{m} \cap \bigcup_{n=1}^{l_{k-1}} f^{-n}\left(U\left(P_{k-1}\right)\right)$ under $f$ may not enter $U\left(P_{k}\right)$ at one iteration precisely, but for simplicity of notations we will assume this is the case. In any event we are faced with at most another finite decomposition of $\phi_{k} F^{m} B_{m}$ into subgraphs identified by which iteration they first enter $U\left(P_{k-1}\right)$.

Hence we assume that $f^{\prime} \phi_{k} F^{m} B_{m} \subset U\left(P_{k-1}\right)$. Since $F^{m} B_{m}$ is the graph of a $C^{1}$ function with slope $\leqslant \omega_{k}$, in $\operatorname{cl} T_{P_{k}} M(\varepsilon)$ we know from Proposition 1 that

$$
\left(\phi_{k-1}^{-1} f^{\prime} \phi_{k} F^{m} B_{m}\right) \cap\left(\mathrm{cl} T_{P_{k-1}} M(\varepsilon)\right)
$$

is a $C^{1}$ fiber bundle over $\phi_{k-1}^{-1} f^{\prime} \phi_{k} F^{m} B_{m}$ intersected with $E_{k-1}^{s}$ in the fundamental neighborhood associated with $W^{s}$ given by $B_{k-1}(\varepsilon)-f\left(B_{k-1}(\varepsilon)\right)$. Furthermore the fibers are the graphs of $C^{1}$ functions from a closed subset of $\mathrm{cl}_{k-1}^{u}(\varepsilon)$ into $\operatorname{cl} E_{k-1}^{s}(\varepsilon)$ and all of the slopes $\leqslant \omega_{k-1}$. By the induction hypothesis the area $\left(\operatorname{dim} E_{k-1}^{u}\right)$ of any fiber is bounded by $K\left(1+\delta^{\prime \prime}\right)^{N-l-m} \exp (-C(N-l-m)$ ) where $\delta^{\prime \prime}$ is between $\delta^{\prime}$ and $\delta$ and $C$ is the appropriate minimum Jacobian. From this we deduce the area(dim $\left.E_{k}^{u}\right)$ of $\phi_{k-1}^{-1} f^{\prime} \phi_{k} F^{m} B_{m}$ by using Fubini's theorem, which applies due to the $C^{1}$ fiber structure. Thus

$$
\operatorname{area}\left(\phi_{k-1}^{-1} f^{\prime} \phi_{k} F^{m} B_{m}\right) \leqslant K\left(1+\delta^{\prime \prime}\right)^{N^{\prime}} \exp \left(-C N^{\prime}\right)
$$

for $N^{\prime}=(N-l-m)$, and where we have redefined $K$. We now translate this back to $\mathrm{cl} T_{k} M(\varepsilon)$ and thus find that for a subgaph of $F^{m} B_{m}$ its length is bounded by

$$
K\left(1+\delta^{\prime \prime}\right)^{N^{\prime}} \exp (-C N)
$$

where again $K$ is redefined to account for bounded factors in the translation, and $N^{\prime}=(N-l-m)$. 
We repeat this procedure to determine the areas of the other subgraphs of $F^{m} B_{m}$ noting that $l$ may change. Since there are finitely many fixed points then the entire area of $F^{m} B_{m}$ is of the form (**) with $K$ modified to account for the number of points and the different $l$. Then translating $(* *)$ back to the initial iteration $n=0$ we immediately get

$$
\operatorname{area}\left(B_{m}\right) \leqslant\left(1+\delta^{\prime}\right)^{m} K\left(1+\delta^{\prime \prime}\right) \exp \left(-C_{k} m-C N^{\prime}\right)
$$

for $N^{\prime}=(N-l-m)$, where $C$ is now the minimum of the $\operatorname{Jac} D f^{u}\left(P_{j}\right)$ for $1 \leqslant j \leqslant k$. We redefine $K$ to get

$$
\operatorname{area}\left(B_{m}\right) \leqslant k\left(1+\delta^{\prime \prime}\right)^{N} \exp (-C N)
$$

and finally arrive at

$$
\begin{aligned}
\operatorname{area}\left(\text { graph } h \cap S_{N}\right) & \leqslant \text { area } A+\text { area } B_{0}+\cdots+\text { area } B_{N-1} \\
& \leqslant(N+1) K\left(1+\delta^{\prime \prime}\right)^{N} \exp (-C N) .
\end{aligned}
$$

Since the exponential decay dominates algebraic growth we amy redefine $K$ to get

$$
\text { area }\left(\text { graph } h \cap S_{N}\right) \leqslant K(1+\delta)^{N} \exp (-C N)
$$

as desired. This completes the proof of Lemma 2.

Applying Lemma 2 we continue the proof of Theorem B. Since $S_{N} \cap H_{v}$ is contained in a linear variety, the area $(\cdot)$, here denoted $\nu^{u}$, is an $r$-dimensional Lebesgue measure. Thus we get

$$
\nu^{u}\left(S_{n} \cap H_{v}\right) \leqslant K(1+\delta)^{N} \exp (-C N) .
$$

Now by application of Fubini's theorem,

$$
\nu\left(S_{N}\right)=\int_{v \in E_{i}^{s}(\varepsilon)}\left[\nu^{u}\left(S_{N} \cap H_{v}\right)\right] d \nu^{s}
$$

or

$$
\begin{aligned}
& \nu\left(S_{N}\right) \leqslant K(1+\delta)^{N} \exp (-C N) \nu^{s}\left(\operatorname{cl} E_{i}^{s}(\varepsilon)\right), \\
& \nu\left(S_{N}\right) \leqslant K(2 \varepsilon) \operatorname{dim} E^{s}(1+\delta)^{N} \exp (-C N) .
\end{aligned}
$$

Then redefine $K$ as $K(2 \varepsilon) \operatorname{dim} E^{s}$; we then get $\nu\left(S_{N}\right) \leqslant K(1+\delta)^{N} \exp (-C N)$. Hence,

$$
\mu\left(U_{N}\left(P_{i}\right)\right) \leqslant K(1+\delta)^{N} \exp (-C N),
$$

where again $K$ is redefined. This completes the proof of Theorem B.

4. The Proof of Theorem A. Let $g=f^{l}$. Then $g$ is a Morse-Smale diffeomorphism with $\Omega(g)=$ Fix $(g)$. We will prove Theorem A for $g$ by induction on $i$ in the quantity $\mu(S(i, i-1, N))$.

From M. Shub [9, part (e), p. 496], we have that there exist $n_{i}$ and $m_{i}$ such that $\left[g^{n_{i}}\left(M_{i}\right)-g^{-m_{i}}\left(\right.\right.$ int $\left.\left.M_{i-1}\right)\right] \subset U\left(P_{i}\right)$. Let $L_{i}=n_{i}+m_{i}$. Then it is evident that $x \in$ $M_{i}$ implies either

$$
g^{n_{i}}(x) \in U\left(P_{i}\right)
$$


or

$$
g^{L_{i}}(x) \in M_{i-1} .
$$

Thus from the definition of $S\left(i, i-1, N+L_{i}\right)$ we have

$$
S\left(i, i-1, N+L_{i}\right) \subset g^{-n_{i}}\left(U_{N+m_{i}}\left(P_{i}\right)\right) \text {. }
$$

Let $i=2$. We consider $S(2,1, N) ; S(2,1, N)=S\left(2,1,\left(N-L_{2}\right)+L_{2}\right)$ so

$$
S\left(2,1,\left(N-L_{2}\right)+L_{2}\right) \subset g^{-n_{2}} U_{N-n_{2}}\left(P_{2}\right) .
$$

Thus by Theorem B,

$$
\mu(S(2,1, N)) \leqslant K^{*} \mu\left(U_{N-n_{2}}\left(P_{2}\right)\right) \leqslant K^{*} K(1+\delta)^{N-n_{2}} \exp \left(-C / l\left(N-n_{2}\right)\right)
$$

for $N \geqslant L_{2}$.

By redefining $K$ we get

$$
\mu(S(2,1, N)) \leqslant K(1+\delta)^{N} \exp (-C / l N) .
$$

Assuming that we have the estimate

$$
\mu(S(i-1,1, N)) \leqslant K(1+\delta)^{N} \exp (-C / l N) \text { for } i<r
$$

consider $S(i, 1, N)$. Then let $A_{N}$ be defined by

$$
S(i, 1, N)=S(i, i-1, N) \cup S(i-1,1, N) \cup A_{N} .
$$

Note. If $x \in A_{N}$ then $x \in M[i, i-1]$ and $f^{N}(x) \in$ int $M_{i-1}$.

Now $\mu(S(i-1,1, N))$ is known by hypothesis and $\mu(S(i, i-1, N))$ is gotten by a trivial modification of the $S(2,1, N)$ case. So we consider $A_{N}$. Next write $A_{N}=D \cup E$, where

$$
\begin{aligned}
& D=\left\{x \in A_{N}: g^{L_{i}}(x) \in M[i, i-1]\right\}, \\
& E=\left\{x \in A_{N}: g^{L_{i}}(x) \in \text { int } M_{i-1}\right\} .
\end{aligned}
$$

Then $g^{L_{i}}(E) \subset S\left(i-1,1, N-L_{i}\right)$ and $E \subset g^{-L_{i}}\left(S\left(i-1,1, N-L_{i}\right)\right)$ so that $\mu(E) \leqslant K^{\prime} \mu\left(S\left(i-1,1, N-L_{i}\right)\right)$, where $K^{\prime}=\max \left\{\operatorname{Jac} g^{-L_{i}}\right\}$ over $M[i, i-1]$. Thus $\mu(E) \leqslant K(1+\delta)^{N} \exp (-C N)$, by modifying $K$.

Finally for $D$ we have $g^{n_{i}} D \subset U\left(P_{i}\right)$ but since any point in $g^{N_{i}} D$ stays out of $M_{i}$ for $N-n_{i}$ iterations we have $g^{n_{i}} D \subset U_{N-n_{i}}\left(P_{i}\right)$. Hence $\mu(D) \leqslant K^{*} \mu\left(U_{N-n_{i}}\left(P_{i}\right)\right)$ or

$$
\mu(D) \leqslant K^{*} K(1+\delta)^{N-n_{i}} \exp \left(-C / l\left(N-n_{i}\right)\right) \text { for } N \geqslant L_{i} .
$$

By redefining $K$, we get

$$
\begin{aligned}
\mu(S(i, l, N)) & \leqslant \mu(S(i, i-1, N))+\mu(S(i-1, j, N))+\mu(D)+\mu(E) \\
& \leqslant K(1+\delta)^{N} \exp (-C / l N)
\end{aligned}
$$

as desired. Since $S(i, j, N) \subset S(i, 1, N)$ this completes the proof for $g$. To extend the result to $f$ we note the following: if $\hat{U}_{m}\left(P_{i}\right)=\left\{x \in U\left(P_{i}\right): f^{k} x \notin M_{1}, k=\right.$ $0,1,2, \ldots, m\}$, then

$$
\hat{U}_{N l}\left(P_{i}\right) \subset U_{N}\left(P_{i}\right)=\left\{x \in U\left(P_{i}\right): g^{k} x \notin M_{1}, k=0,1, \ldots, N\right\} .
$$




$$
\begin{aligned}
& \text { Hence } \mu\left(\hat{U}_{N}\left(P_{i}\right)\right) \leqslant K(1+\delta)^{N} \exp (-C N) \text { or } \\
& \qquad \mu\left(U_{N}\left(P_{i}\right)\right) \leqslant\left[K(1+\delta)^{1 / l}\right](1+\delta)^{N} \exp (-C / l) N .
\end{aligned}
$$

The proof is finished by noting that $C / l$ is the minimum of the quantities $\left\{1 / m \log \mathrm{Jac} D_{p_{j}} f^{m} \mid W^{u}\left(p_{j}\right)\right\}$ where the minimum is taken over all $p_{j}$ in the nonwandering set for $f$ which are not sinks and $f^{m} p_{j}=p_{j}$.

5. Morse-Smale flows. The goal of this section is to prove a corresponding theorem for Morse-Smale flows.

For $x$ a fixed point of a flow $\phi_{t}$ we define

$$
\phi^{u}(x)=\log \mathrm{Jac} D \phi_{1} \mid E_{x}^{u} \rightarrow E_{x}^{u} .
$$

For $\gamma$ a closed orbit of $\phi_{t}$ with period $\tau$ we define

$$
\phi^{u}(\gamma)=1 / \tau\left(\min _{y \in \gamma}\left\{\log \mathrm{Jac} D \phi_{\tau} \mid E_{y}^{u} \rightarrow E_{y}^{u}\right\}\right) .
$$

A fixed point $x$ or a closed orbit $\delta$ is an attractor of the flow $\phi_{t}$ if there is an open set $U$ containing it satisfying $\phi_{t}(U) \subset U$ for all $t \geqslant 0$, and

$$
\bigcap_{t \geqslant 0} \phi_{t}(U)=\left\{\begin{array}{l}
\{\gamma\}, \\
\{x\} .
\end{array}\right.
$$

Definition. Let $X$ be a vector field on $M$, and denote by $\phi_{t}$ its induced flow. We say $\phi_{t}$ is a Morse-Smale flow if $X$ satisfies the following conditions [6]:

(a) $\Omega(X)$ is the union of a finite number of fixed points $x_{1}, x_{2}, \ldots, x_{m}$ and a finite number of closed orbits $\gamma_{1}, \gamma_{2}, \ldots, \gamma_{n}$ of $X$.

(b) The $x_{i}, \gamma_{j}$, are all hyperbolic.

(c) The stable and unstable manifolds of the $x_{i}, \gamma_{j}$ have transversal intersection.

THEOREM C. Let $\phi_{t}$ be a Morse-Smale flow on M. There is a filtration $\mathscr{M}=\left\{M_{i}\right\}_{i=1}^{r}$ such that:

(a) All attractors for $\phi_{t}$ are in $M_{1}$.

(b) For all $\delta>0$,

$$
\mu(S(i, 1, T)) \leqslant K(1+\delta)^{T} \exp (-C T)
$$

for $i>2, K>0$ independent of $T$, and $C=\min _{x_{j}, \gamma_{j}}\left\{\phi^{u}\left(x_{j}\right), \phi^{u}\left(\gamma_{j}\right)\right\}$ where $x_{j}, \gamma_{j}$ are, respectively, the fixed points and closed orbits of $\phi_{t}$ which are not sinks or attractors.

Proof. We show how to modify our previous arguments to this case.

If $x$ is a fixed point of $\phi_{t}$, then our previous work suffices to describe the behavior of trajectories in the neighborhood of $x$ by considering the time one diffeomorphism $g=\phi_{1}$.

If $\gamma$ is a closed orbit of $\phi_{t}$ which is not an attractor, then $T_{\gamma} M$ splits continuously into $E+E^{s}+E^{u}$ where $E$ is the one-dimensional bundle tangent to the flow and dimension $E^{u} \geqslant 1$. For $g=\phi_{\tau}$ the time $\tau$ diffeomorphism where $\tau$ is the period of $\gamma$, $\gamma$ is a closed, infinite, nonhyperbolic set in $\Omega(g)$. However, since $D g(y)$ expands $E_{y}^{u}$ more rapidly that $E_{y}$ for any $y \in \gamma$, then given $\varepsilon>0$ we can find $\delta, 0<\delta<\varepsilon$, so that if $g^{k}(x) \in B_{y}(\varepsilon)$ for $0 \leqslant k \leqslant n$ but $g^{n+1}(x) \notin B_{y}(\varepsilon)$, then $d\left(g^{N+1}(x), \gamma\right)>\delta$. By compactness we can cover $B_{\gamma}(\delta)$ by a finite number of $\left\{B_{y}(\varepsilon)\right\}_{y \in \gamma}$. 
The effect of this construction is to permit use of the previous established volume and area estimates in the neighborhood of $\gamma$ by considering only a finite number of neighborhoods covering $\gamma$. If $N$ is a $C^{1}$ submanifold of $M$ in $\operatorname{cl} B \gamma(\delta)$, then $N$ decomposes into a finite number of submanifolds determined by its intersections with $B_{y}(\varepsilon)$. Let $N_{y}$ be a typical one. Then $\phi_{y}^{-1} N_{y}$ is a $C^{1}$ submanifold of $T_{y} M(\varepsilon)$. If, in addition, it is the graph of a $C^{1}$ function of a closed subset of $E_{y}^{u}(\varepsilon)$ into $E_{y}^{c s}(\varepsilon)=E_{y}^{s}(\varepsilon)+E_{y}(\varepsilon)$ with prescribed slope, then under iteration by $G=$ $\phi_{y}^{-1} \circ g \circ \phi_{y}$, it remains the graph of a $C^{1}$ function of the unstable space $E_{y}^{u}$ into the center stable space $E_{y}^{c s}$. That is the graph transform properties from before apply here [2]. Locally $\gamma$ behaves like a finite number of nonwandering points as far as the area lemma (Lemma 2) is concerned.

The only other issue is that of transversal intersection. The stable and unstable manifold of any two closed orbits, including fixed points, have transversal intersection. In the case of a closed orbit there is a third direction-the flow direction. We actually need each unstable manifold to intersect each center stable manifold transversally. This occurs since the stable and center stable manifolds intersect along the flow. Hence the analogue of Proposition 1 is true for Morse-Smale flows. Also by our previous remark the area lemma, Lemma 2, has an analogue. We thus prove our volume estimates locally using fixed time diffeomorphisms $g=\phi_{\tau}$ and finish the proof by normalizing the estimates by dividing the exponential constants by $\tau$ when necessary.

\section{BIBLIOGRAPHY}

1. R. Bowen, Equilibrium states and the ergodic theory of Anosov diffeomorphisms, Lecture Notes in Math., vol. 470, Springer-Verlag, Berlin and New York, 1975.

2. R. Bowen and D. Ruelle, The ergodic theory of Axiom A flows, Invent. Math. 29 (1975), 181-202.

3. R. Bowen, Periodic orbits of hyperbolic flows, Amer. J. Math. 94 (1972), 1-37.

4. __ A horseshoe with positive measure, Invent. Math. 29 (1975), 203-204.

5. D. Fried and Michael Shub, Entropy, linearity, and chain recurrence, Extrait des Publications Mathematiques, No. 50, 1978, pp. 203-214.

6. J. Palis, On Morse-Smale dynamical systems, Topology, Vol. 8, Pergamon Press, New York and Oxford, 1969, 385-405.

7. J. Palis and S. Smale, Structural stability theorems, Proc. Sympos. Pure Math., Vol. 14, Amer. Math. Soc., Providence, R.I., 1970, pp. 223-232.

8. C. Pugh and M. Shub, The S-stability theorem for flows, Invent. Math. 11 (1970), 150-158.

9. M. Shub, Stability and genericity for diffeomorphisms, Dynamical Systems (M. Peixoto, ed.), Academic Press, New York, 1973, pp. 493-514.

10. S. Smale, Differentiable dynamical systems, Bull. Amer. Math. Soc. 71 (1967), 747-814.

11. H. Wisniewski, Rate of approach to minima and sinks - the $C^{2}$ Axiom A no cycles case. Geometric Dynamics (Proc. Internat. Sympos. in Dynamical Systems, Rio de Janiero, Brazil, 1981), Lecture Notes in Math., vol. 1007, Springer-Verlag, Berlin and New York.

Division of Research, School of Business, Seton Hall University, South Orange. New JERSEY 07079 . 